\title{
Innovative and Useful Approaches on Trace Determinations of Organic and Inorganic Analytes
}

\section{Ulusoy HI}

\author{
Faculty of Pharmacy, Department of Analytical Chemistry, Cumhuriyet University, Sivas, Turkey
}

\begin{abstract}
Sample pretreatment procedures is one of the most important steps in analysis of complex samples. Even a research laboratory has complicated or sensitive instruments, it is not easy to obtain reliable results if all variables can be considered carefully in your samples. All over the world, most of analytical chemists try to find new methods and develop new materials for sensitive and correct analysis of target species. Prior to analysis in instrumental tools, sample preparation is a critical step affecting all results. Accuracy and reliability of results are directly affected by these steps. An ideal pretreatment procedure should be simple, cost effective, environmental friendly and compatible detection method. There are two fundamental goal of pre-treatment procedures; separation of matrix components and pre-concentration of trace analyte species. If a method can accomplish one of these goals, a conventional analysis approaches will be more sensitive or selective for trace analyte species. This paper includes a few useful approaches of our research group for trace determination of organic and inorganic species. Each of method focus on a target ion or molecule by using a pre-concentration method such as cloud point extraction (CPE), solid phase extraction (SPE), and magnetic solid phase extraction (MSPE), fabric phase sorptive extraction (FPSE). Application of developed method was carried out in various complex samples including food, cosmetic, water and other environmental samples.
\end{abstract}

Keywords: Trace analysis; Pre-concentration and separation methods; Chromatography; Spectroscopy

\section{INTRODUCTION}

Trace analysis is a chemical term which mean analysis of organic and inorganic species at trace level. Mostly, these kind of analysis need complicated instruments or a pre-treatment procedure. Matrixes of samples are so complicated to determine trace organic or inorganic molecules. Main objectivities of this type analysis are trace levels of target species and complex ingredients of samples. In a lot of routine analysis laboratory, generally directly determinations are carried out without any pre-treatment procedures. Because the concentration of target molecules or ions is comparably high, it is not generally need a pre-concentration and separation step. Detection limit and selectivity of most instrumental system are enough for major components of samples. However, especially toxicological analysis need more consideration and sensitivity. Concentration of target molecules or ions in these type samples is so low for directly determination and these samples includes a lot of interferences.

Separation and pre-concentration technical are focused on two main objectivities. To Increase concentration of target species until detection limit of determination system, to remove interference ion or molecules from sample medium (sometimes, to remove from analyte from sample medium). If this procedure can be carried out successively, it is difficult the reliable results for each sample type.

A classical sample pretreatment procedure includes two main step. Increasing of analyte concentration to detection limit of method and separation of matrix species due to possible interfering effect. In our research group, we developed a lot of method for organic and inorganic species at trace levels. Mainly, the methods developed by our group are based on kinetic analysis, cloud point extraction, solid phase extraction, magnetic solid phase extraction; polymer based solid phase extraction, etc.

Any analytical determination methodology like spectroscopy, chromatography, and electroanalytical approaches can be used after a pre-concentration technical was optimized in detail. Generally, the obtained final phase is suitable for one of these methods. But, chromatographically methods like HPLC and GC and spectroscopic ally methods AAS and molecular spectroscopy.

\section{LITERATURE REVIEW}

The developed methods for trace organic and inorganic analyte

In this section, a few examples of studies about trace determination were given with other details. Nitrate and nitrite determination by

Correspondence to: Halil İbrahim Ulusoy, Faculty of Pharmacy, Department of Analytical Chemistry, Cumhuriyet University, 58140, Sivas, Turkey, Tel: +90 34621910 10/3905; E-mail: hiulusoy@yahoo.com

Received: January 28,2019; Accepted: February 20, 2019; Published: February 27, 2019

Citation: Ulusoy HI (2019) Innovative and Useful Approaches on Trace Determinations of Organic and Inorganic Analytes. Pharm Anal Acta 10:606. doi: $10.35248 / 2153-2435.19 .10 .606$

Copyright: (C) 2019 Ulusoy HI. This is an open-access article distributed under the terms of the Creative Commons Attribution License, which permits unrestricted use, distribution, and reproduction in any medium, provided the original author and source are credited. 
kinetic analysis method was carried out by using a simple UV-VIS spectrophotometry [1]. It can be considered the classical detection principle for the determination of nitrite. The most widely used methods for the determination of nitrite are based on the wellknown Griess reaction. Nitrite reacts with a primary aromatic amine to form a diazonium salt. The absorbance change can be easily followed by spectrophotometric ally [1]. These inorganic anions are extremely important in biological and environmental studies. A simple, sensitive and rapid kinetic spectrophotometric method for the determination of nitrite in different natural and wastewater samples is presented in the developed method. The reaction is initiated by adding the known volumes of a nitrite ion solution of identified concentration to acid solutions of Cresyl violet perchlorate, which the color of indicator reagent changes to yellow with time. Then, the absorbance changes are monitored spectrophotometric ally at $\lambda \max =585 \mathrm{~nm}$ for the concentration range of $0.01-2 \mu \mathrm{g} \mathrm{mL}^{-1}$ and at $\lambda \max =409 \mathrm{~nm}$ for the concentration range of $0.5-5 \mu \mathrm{g} \mathrm{mL} \mathrm{m}^{-1}$ of nitrite ion at $40^{\circ} \mathrm{C}$. The method was successfully applied to the determination of nitrite in various natural samples.

The use of cloud point extraction offers (CPE) an alternative to conventional extraction systems. Aqueous solutions are used in the CPE method instead of toxic and flammable organic solvents. In addition, CPE offers higher recovery efficiency and a large preconcentration factor. An image for steps of CPE can be shown in Figure 1. As good application of this methodology can be given for selenium species. They are also very important for living organisms. A useful pre-concentration and determination method was proposed for trace selenium in food samples [2]. The procedure is based on complex formation of Pyronine B with Se (IV) ions in the presence of sodium dodecyl sulphate (SDS) and Ponpe 7.5. The variables affecting complex formation, extraction and phase separation were studied and optimized. Under the experimental conditions used, the calibration graph was linear in the range of 20-1700 $\mathrm{ng} \mathrm{mL}^{-1}$ for Se (IV) ions. The limit of detection was $3.81 \mathrm{ng}$ $\mathrm{mL}^{-1}$ of Se (IV) and the relative standard deviation for 5 replicate

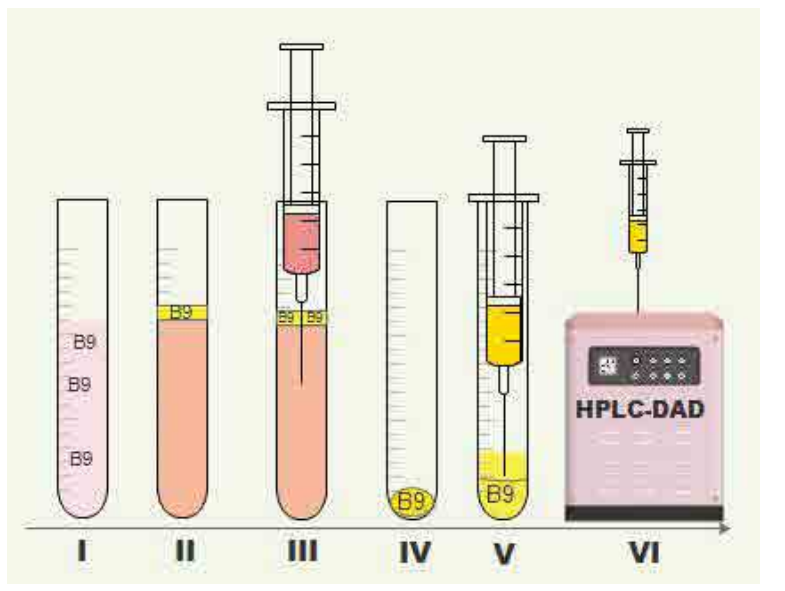

Figure 1: Steps of cloud point extraction [3].

I) Model Solution before CPE.

II) Separation of surfactant rich phase (SRP) by centrifugation.

III) Removal of aquatic phase.

IV) SRP on the bottom of tube.

V) Dilution of SRP with a suitable solvent.

VI) Determination of target species by an instrument. determinations at $250 \mathrm{ng} \mathrm{mL}^{-1}$ concentration level was $2.45 \%$. The method was successfully applied to the determination of total selenium in some food samples and alcoholic and nonalcoholic beverages.

Cloud point extraction as useful methodology is based on a property of most nonionic surfactants in aqueous solutions and becoming turbid solution when being heated to the particular temperature, which is known as cloud point temperature (Tc). A sample solution including organic molecules are extracted into surfactant-rich phase. Vitamins molecules are ideal target groups for CPE. They are important essentially organic compounds for humans. A fast, efficient, cost-effective, and environmental-friendly analytical methodology was developed for pre-concentration and determination of trace folic acid, is known as vitamin B9, in food samples prior to high performance liquid chromatography with diode array detection (HPLC-DAD) [3]. The method is based on formation of stable complexes between folic acid and $\mathrm{Fe}$ (III) ions at $\mathrm{pH}$ 8.0. The formed complexes were extracted to a nonionic surfactant phase containing PONPE 7.5. The limit of detection (LOD) of folic acid was $6.06 \mathrm{ng} \mathrm{mL}^{-1}$, the linear range of quantitation for folic acid was $20-1200 \mathrm{ng} \mathrm{mL}^{-1}$ and the correlation coefficients of the calibration curves were 0.9976 . After validation of the method was carried out, the method was applied to the determination of folic acid in food samples, including baby foods, vegetables, cereals, and pharmaceutical samples.

Solid phase extraction (SPE) methods are mainly preferred in sample preparation owing to useful and practical properties. Use of magnetic solids in SPE methods provided important advantages to overcome these complications. Their magnetic properties help easy separation using a magnet, after accumulation of target molecules on particles. Experimental steps of magnetic solid phase extraction (MSPE) was given in Figure 2. In an application, synthetic drug active ingredients are also followed by international authorized organizations. Imitation or fake products are sold in everywhere. So, determination of drug molecules at trace levels is a challenge for analytical chemists. A sensitive analytical methodology was investigated to concentrate and determine of sildenafil citrate (SLC) present at trace level in herbal supplementary products [4]. The proposed method is based on simple and sensitive preconcentration of SLC by using magnetic solid phase extraction with new developed magnetic nanodiamond/graphene oxide hybrid ( $\left.\mathrm{Fe}_{3} \mathrm{O}_{4} @ \mathrm{ND} @ \mathrm{GO}\right)$ material as a sorbent. Experimental variables affecting the extraction efficiency of SLC like; $\mathrm{pH}$, sample

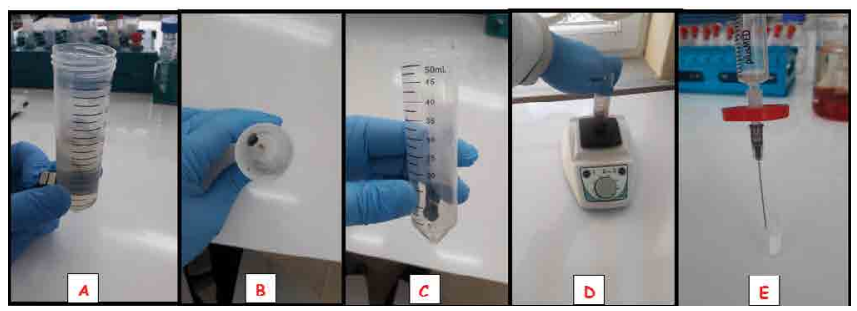

Figure 2: Main steps of MSPE.

A) Separation of magnetic particles by an external magnet.

B) Removal of aquatic phase.

C) Remaining phase on bottom of tube.

D) Desorption of adsorbed molecules by a vortex.

E) Filtration of pre-concentrated samples before determination step. 
volume, eluent type and volume, extraction time and amount of adsorbent were studied and optimized in detail. Under optimized conditions, linear range was ranged from 5.00 to $250.00 \mathrm{ng} \mathrm{mL}^{-1}$ with $\mathrm{R}^{2}$ of 0.9952 . The limit of detection (LOD) was $1.49 \mathrm{ng} \mathrm{mL}^{-1}$ and the recoveries at two spiked levels were ranged from 94.0 to $104.1 \%$ with the relative standard deviation (RSD)<7.1\% $(n=5)$. The enhancement factor (EF) was 86.9.

An another application of cloud point extraction was applied for mercury ions. Toxic metals are potential dangerous elements for environment and human health. Sensitive and correct determinations of these metals draws great attention. A new micelle-mediated separation and pre-concentration method was developed for ultra-trace quantities of mercury ions prior to spectrophotometric determination [5]. The method is based on cloud point extraction (CPE) of $\mathrm{Hg}$ (II) ions with polyethylene glycol tert-octylphenyl ether (Triton X-114) in the presence of chelating agents such as 1-(2-pyridylazo)-2-naphthol (PAN) and 4-(2-thiazolylazo) resorcinol (TAR). Hg (II) ions react with both PAN and TAR in a surfactant solution yielding a hydrophobic complex at $\mathrm{pH} 9.0$ and 8.0, respectively. The phase separation was accomplished by centrifugation for $5 \mathrm{~min}$ at $3500 \mathrm{rpm}$. The calibration graphs obtained from $\mathrm{Hg}$ (II)-PAN and $\mathrm{Hg}$ (II)-TAR complexes were linear in the concentration ranges of 10-1000 $\mathrm{g} \mathrm{L}^{-1}$ and $50-2500 \mathrm{~g} \mathrm{~L}^{-1}$ with detection limits of 1.65 and $14.5 \mathrm{~g}$ $\mathrm{L}^{-1}$, respectively. The relative standard deviations (RSDs) were $1.85 \%$ and $2.35 \%$ in determinations of 25 and $250 \mathrm{~g} \mathrm{~L}^{-1} \mathrm{Hg}$ (II), respectively. The developed methods were successfully applied to determine mercury concentrations in environmental water samples. In another method for mercury ions: the cloud point extraction method was based on the selective formation of a complex between selenium (IV) ions and 4,5-diamino-6-hydroxy2-mercapto pyrimidine (DAHMP) [6]. Then, the formed stable complex was extracted to a nonionic surfactant phase of Triton X-114. After phase separation was carried out via decantation, the surfactant-rich phase was diluted with $20 \%$ ethanol solution and the absorbance signal was measured at $458 \mathrm{~nm}$ using an UVVIS spectrophotometer. Under the optimized conditions, linear calibration curves were obtained over a range of $20-1500 \mathrm{ng} \mathrm{mL}^{-1}$ with detection and quantification limits of $6.06 \mathrm{ng} \mathrm{mL}^{-1}$ and 19.89 $\mathrm{ng} \mathrm{mL} \mathrm{m}^{-1}$, respectively. The relative standard deviation was $2.80 \%$ for 5 replicate measurements at $250 \mathrm{mg} \mathrm{L}^{-1}$ concentration level.

In the determination of another metallic ion, molybdenum, cloud point extraction (CPE) was used for the pre-concentration of molybdenum (Mo), after the formation of a complex with Victoria pure blue $\mathrm{BO}(\mathrm{VPB}+)$ in presence of excess thiocyanate

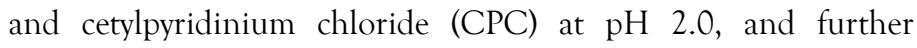
determination by flame atomic absorption spectrometry (FAAS) using Triton X-114 as surfactant [7]. The main factors affecting the $\mathrm{CPE}$, such as concentration of thiocyanate, $\mathrm{VPB}+, \mathrm{CPC}$, Triton $\mathrm{X}-114, \mathrm{pH}$, equilibration temperature and incubation time, were optimized for the best extract efficiency. Under the optimized conditions, a 32-fold enhancement factor was reached. The lower limit of detection (LOD) obtained under the optimal conditions was $2.18 \mathrm{ng} \mathrm{L}^{-1}$. The accuracy (REs) and precision (RSDs) for five replicate determinations at $250 \mathrm{ng} \mathrm{L}^{-1}$ Mo were 3.25 and $2.36 \%$ after pre-concentration with CPE. The method was successfully applied to the determination of Mo in beverages and food samples and in certified reference materials.

Radioactive elements have great hazards on environment and human health. Determination of trace uranyl ions was performed by using mixed micellar system and spectrophotometric determination [8]. The method is based on cloud point extraction of uranyl ions after formation of an ion-association complex in the presence of Celestine Blue and sodium dodecyl sulfate. Then, the formed complex was extracted to non-ionic surfactant phase of Triton X-114 at $\mathrm{pH}$ 8.0. The optimal extraction and reaction conditions (e.g., concentrations and types of surfactants, concentration of complex forming agent, incubation conditions) were studied and analytical characteristics of the method (e.g., limit of detection, linear range, pre-concentration factor) were obtained by experimental studies. Linearity was obeyed in the range of 50 $1.500 \mathrm{ng} \mathrm{mL}^{-1}$ for uranium (VI) ion and the detection limit of is $14.20 \mathrm{ng} \mathrm{mL}^{-1}$. The method was applied to the determination of uranium (VI) in several environmental samples.

Almost each species of arsenic ions is highly toxic for human health. There are a lot of methodology for their sensitive determinations. In a method, cloud point extraction (CPE) methodology has successfully been employed for the pre-concentration of ultra-trace arsenic species in aqueous samples prior to hydride generation atomic absorption spectrometry (HGAAS) [9]. As (III) has formed an ion-pairing complex with Pyronine $\mathrm{B}$ in presence of sodium dodecyl sulfate (SDS) at $\mathrm{pH} 10.0$ and extracted into the non-ionic surfactant, polyethylene glycol tert-octylphenyl ether (Triton X-114). After phase separation, the surfactant-rich phase was diluted with $2 \mathrm{~mL}$ of $1 \mathrm{M} \mathrm{HCl}$ and $0.5 \mathrm{~mL}$ of $3.0 \%(\mathrm{w} / \mathrm{v})$ Antifoam A. Under the optimized conditions, a pre-concentration factor of 60 and a detection limit of $0.008 \mathrm{ng} \mathrm{mL}{ }^{-1}$ with a correlation coefficient of 0.9918 were obtained with a calibration curve in the range of 0.03-4.00 $\mathrm{ng} \mathrm{mL} \mathrm{mL}^{-1}$. The proposed preconcentration procedure was successfully applied to the determination of As (III) ions in certified standard water samples and some samples including natural drinking water and tap water samples.

A cloud point extraction (CPE) method has been developed for the pre-concentration of trace aluminum prior to its determination by flame atomic absorption spectrometry (FAAS) [10]. The CPE method is based on the complex of Al (III) with Xylidyl Blue (XB) and then entrapped in non-ionic surfactant Triton X-114. The main factors affecting CPE efficiency, such as $\mathrm{pH}$ of sample solution, concentration of $\mathrm{XB}$ and Triton X-114, equilibration temperature and time, were investigated in detail. An enrichment factor of 50 was obtained for the preconcentration of Al (III) with $50 \mathrm{~mL}$ solution. The proposed method has been applied for determination of trace amount of aluminum in mineral water samples with satisfactory results.

Tin is a toxic metal, which can accumulate in the human body and in animal tissue. Determination of tin species in food samples is very important in order to follow the transition of dissolved tin species in canned foods. For Sn analysis, a new cloud point extraction procedure was developed for the separation and preconcentration of tin ions in water and food samples. The method is based on the complexation of Sn (IV) ions with calcon carboxylic acid (CCA) in the presence of the non-ionic surfactant [11]. The enriched analyte ions were determined by flame atomic absorption spectrometry. The proposed method is very versatile and economic, because it is used conventional FAAS. A pre-concentration factor of 100 and detection limit of $2.86 \mathrm{ng} \mathrm{mL}^{-1}$ were obtained from the calibration curve constructed in the range of $10-1300 \mathrm{ng} \mathrm{mL}^{-1}$ with a correlation coefficient of 0.9978 . The method was successfully applied for determination of tin ions in certified reference materials, natural spring water, canned foods and fruit juices. 


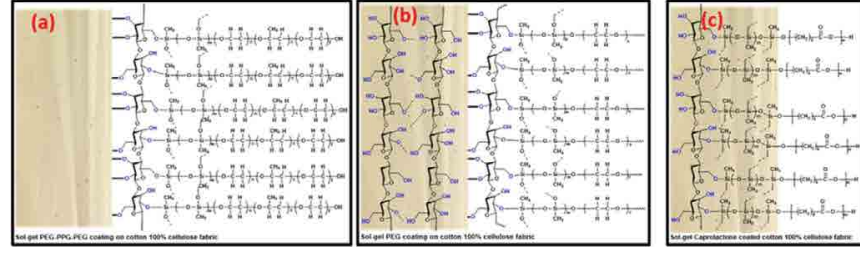

Figure 3: Schematic representation of (a) sol-gel PEG-PPG-PEG; (b) sol-gel CW 20M, and (c) sol-gel Polycaprolactone coated FPSE media.

Cobalt is an essential trace element present in most body tissues. Cobalt toxicity is lower than other metals. However, at high concentrations cobalt can cause several health problems such as asthma and skin irritation and systemic allergic dermatitis. A new method has been developed for pre-concentration of cobalt at trace levels in beverage samples using calcon carboxylic acid as chelating agent and cetyl pyridinium chloride as an auxiliary ligand and entrapped into Triton X-114 prior to its determination by flame atomic absorption spectrometry (FAAS) [12]. The main parameters affecting cloud point extraction (CPE) efficiency such as $\mathrm{pH}$, concentration of the complexing agent, cationic and nonionic surfactant concentration, salt effect, the equilibrium time, and temperature were investigated and optimized. After optimization of the CPE conditions, a preconcentration factor of 60 , an enhancement factor of 106 , and a detection limit of $0.20 \mathrm{ng} \mathrm{mL}^{-1}$ by $\left(R^{2}=0.9978\right)$ were obtained from a calibration curve constructed in the range of $0.7-100 \mathrm{ng} \mathrm{mL}^{-1}$.

Recently, a new and simple methodology has been proposing by Kabir et al. for sensitive determination of organic molecules. It was named as Fabric Phase Sorptive Extraction (FSPE) by developers. It is based on selective modification of a fabric surface for target molecules. The most important advantages of this method are very easy separation of solid phase after interaction and very fast experimental procedures. An illustration of various fabric phases was shown in Figure 3. This method was applied for sensitive determination of described antitumor drugs in biological matrices. The method describes a fast, sensitive and selective procedure for target molecules by high performance liquid chromatography (HPLC) in human whole blood, plasma, and urine samples succeeding an extraction by innovative fabric phase sorptive extraction (FPSE) [13]. The linear ranges for each drug was obtained using working solutions in the concentration range 0.025-10.0 $\mu \mathrm{g} /$ $\mathrm{mL}$, and the analyses were carried out in six replicate for each concentration.

\section{DISCUSSION AND CONCLUSION}

This paper presents a few examples about trace determination of organic and inorganic species. Every day, new approaches have been presenting by scientist based on cloud point extraction, solid phase extraction or other extraction methods. All of these approaches aim to find better methods for sensitivity and selectivity. But, simplicity and costs for experimental procedures are also important as analytical parameters. Because, most of routine analysis laboratories have limited facilities and chemicals. So, new approaches by using cheap equipment and chemicals are preferred for routine analysis.

\section{REFERENCES}

1. Gürkan R, Ulusoy HI, Akçay M. A new analytical method for the kinetic spectrophotometric determination of trace amounts of nitrite in some environmental water samples. Eurasian J Anal Chem. 2010;5:16-31.

2. Ulusoy HI, Yilmaz O, Gürkan R. A micellar improved method for trace levels selenium quantification in food samples, alcoholic and nonalcoholic beverages through CPE/FAAS. Food Chem. 2013;139:1008-1014.

3. Ulusoy S, Acidereli H, ErdoПan S, Ulusoy HI. A new approach to the determination of folic acid at trace levels: Using a Fe(iii)-folic acid complex to amplify analytical signal. RSC Adv. 2016;6:40115-40122.

4. Yilmaz E, Ulusoy HI, Demir O, Soylak M. A new magnetic nanodiamond/graphene oxide hybrid (Fe3O4@ND@GO) material for pre-concentration and sensitive determination of sildenafil in alleged herbal aphrodisiacs by HPLC-DAD system. J Chromatogr B Anal Technol Biomed Life Sci. 2018; 1084:113-121.

5. Ulusoy HI, Gürkan R, Ulusoy S. Cloud point extraction and spectrophotometric determination of mercury species at trace levels in environmental samples. Talanta. 2012;88:516-523.

6. Ulusoy HI. Simple and useful method for determination of inorganic selenium species in real samples based on UV-VIS spectroscopy in a micellar medium. Anal Methods. 2015;7:953-960.

7. Gürkan R, Aksoy U, Ulusoy HI, Akçay M. Determination of low levels of molybdenum (VI) in food samples and beverages by cloud point extraction coupled with flame atomic absorption spectrometry. J Food Compos Anal. 2013;32:74-82.

8. Ulusoy HI. Determination of trace uranyl ions in aquatic medium by a useful and simple method. J Radioanal Nucl Chem. 2014;302:497-504.

9. Ulusoy HI, Akçay M, Ulusoy S, Gürkan R. Determination of ultra trace arsenic species in water samples by hydride generation atomic absorption spectrometry after cloud point extraction. Anal Chim Acta. 2011;703:137-144.

10. Ulusoy HI, Gürkan R, Aksoy U, Akçay M. Development of a cloud point extraction and preconcentration method for determination of trace aluminum in mineral waters by FAAS. Microchem J. 2011; 99:76-81.

11. Ulusoy S, Ulusoy HI, Akçay M, Gürkan R. Inexpensive and versatile method for trace Sn (II) and Sn (IV) ions in food samples by CPE/ FAAS. Food Chem. 2012;134:419-426.

12. Ulusoy HI, Gürkan R, Demir O, Ulusoy S. Micelle-mediated extraction and flame atomic absorption spectrometric method for determination of trace cobalt ions in beverage samples. Food Anal Methods. 2012;5:454-463.

13. Locatelli M, Tinari N, Grassadoni A, Tartaglia A, Macerol D, Piccolantonio S, et al. FPSE-HPLC-DAD method for the quantification of anticancer drugs in human whole blood, plasma, and urine. J Chromatogr B. 2018;1095:204-213. 